\title{
Genetically modified tumour vaccines producing IL-12 augment chemotherapy of HPV16-associated tumours with gemcitabine
}

\author{
ROMANA MIKYŠKOVÁ, MARIE INDROVÁ, JANA ŠÍMOVÁ, \\ JANA BIEBLOVÁ, JAN BUBENÍK and MILAN REINIŠ
}

\author{
Institute of Molecular Genetics v.v.i., Academy of Sciences of the Czech Republic, Prague 142 20, Czech Republic
}

Received December 9, 2010; Accepted February 16, 2011

DOI: 10.3892/or.2011.1221

\begin{abstract}
Genetically modified tumour cells producing cytokines such as interleukin 12 (IL-12) are potent activators of the antitumour immune responses and represent a promising therapeutic modality when combined with chemotherapy. The objective of this study was to examine whether IL-12-producing cellular vaccines can augment chemotherapy of human papilloma virus (HPV) 16-associated murine tumours with the cytostatic agent gemcitabine (GEM). We found that peritumoral administration of IL-12-producing tumour vaccines enhanced the effect of cytoreductive therapy with GEM both in non-metastasizing murine carcinoma TC-1 and in metastasizing murine carcinoma MK16. The percentage of mice with MK16 metastases and the number of lung metastatic nodules was substantially decreased. In another clinically relevant setting, surgical minimal residual tumour disease, the administration of IL-12-producing tumour vaccine and GEM after the MK16 tumour surgery reduced the percentage of mice with tumour recurrences; similarly, the percentage of metastasis-bearing mice and the number of metastatic nodules was decreased. Tumour inhibitory effects exerted by GEM plus IL-12 were associated with high production of interferon- $\gamma$ (IFN $\gamma)$ by splenocytes. Our results suggest that the IL-12-producing vaccine can enhance the effect of GEM chemotherapy in some HPV16-associated murine tumour models.
\end{abstract}

\section{Introduction}

Cancer immunotherapy is an attractive approach to cancer treatment. It has been suggested that immunotherapy has the capacity to achieve better effectiveness when used in combination with chemotherapy. Synergistic effects of combi-

Correspondence to: Dr Milan Reiniš, Institute of Molecular Genetics v.v.i., Academy of Sciences of the Czech Republic, Vídeňská 1083, Prague 4, 142 20, Czech Republic

E-mail: milan.reinis@img.cas.cz

Key words: gemcitabine, human papilloma virus, interleukin 12, lung metastases, cellular vaccines, chemoimmunotherapy nations of immunotherapy and chemotherapy have been shown in a number of pre-clinical and clinical studies (1-8). Chemotherapeutic drugs affect rapidly growing cells, and as a consequence cause collateral damage to cells of the immune system. In this regard they are considered immunosuppressive. However, there is increasing evidence that some cancer chemotherapies may actually aid the immunotherapy by activating the immune system rather than suppressing it (9-11). Chemotherapeutic drugs such as cyclophosphamide, doxorubicin, and paclitaxel were reported to have immunomodulatory activities and appeared to be suitable for chemoimmunotherapy $(12,13)$.

Gemcitabine (GEM), a pyrimidine nucleoside anti-metabolite, is a relatively recently developed cytostatic agent with potent antitumour activity demonstrated in a wide spectrum of in vitro and in vivo animal tumour models and its efficacy was confirmed in a variety of clinical settings (14-16). It has been shown that GEM has also the immunomodulatory properties. Nowak et al $(3,10,11)$ reported on a series of studies suggesting that GEM might be particularly useful when used in combination with immunotherapy because it was able to induce tumour necrosis/apoptosis while not adversely affecting $\mathrm{T}$ cell function. In addition to its pro-apoptotic effects, GEM selectively promotes the cell-mediated immune response over the humoral immune response by selectively inhibiting B-cell proliferation, decreasing memory $\mathrm{T}$ cells, and promoting the activation of naive $\mathrm{T}$ cells and function of $\mathrm{CD}^{+} \mathrm{T}$ cells (17-19). Pre-treatment with GEM can enhance the antigenicity and immunogenicity of tumours by promoting adaptive immune responses (19). GEM was successfully used to reduce the percentage of myeloid derived suppressor cells (MDSCs) in the spleen and in the tumour microenvironment, but did not reduce $\mathrm{CD}^{+}{ }^{+}$and $\mathrm{CD}^{+}{ }^{+} \mathrm{T}$ cells, natural killer $(\mathrm{NK})$ cells, macrophages and B cells (18-21).

IL-12 is a potent immunostimulatory cytokine exerting antitumour effects in several animal models (22-25) (reviewed in refs. 4,8,26). The broad antitumour activity of IL-12 is related to its ability to induce Th1 type responses and to activate NK cells, natural killer T cells, cytotoxic T lymphocytes (CTLs) and antigen-presenting cells (27-29). Furthermore, IL-12 is an anti-angiogenic agent which can antagonize proangiogenic signals during the tumour development $(28,30)$. To achieve significant antitumour effects, high systemic doses of cytokines are usually required. Serious toxicity 
associated with the systemic administration of the high doses was observed in humans. Therefore, local peritumoral gene therapy has been proposed as a suitable strategy to ensure the required levels of cytokine in the peritumoral milieu and low levels in the circulation (30).

In our previous studies, the efficacy of the recombinant IL-12 or cellular vaccines engineered to produce IL-12 in the treatment of early tumour transplants of HPV16-associated tumours, as well as in the treatment of tumour recurrences after surgery or cytoreductive chemotherapy was demonstrated $(5-7,31)$.

In this report, we have examined the local treatment with IL-12-producing tumour cellular vaccine and we have shown that this vaccine augments the antitumour effects of chemotherapy with cytostatic agent GEM, using HPV16-associated murine tumours differing in major histocompatibility complex (MHC) class I cell-surface expression and ability to metastasize.

\section{Materials and methods}

Mice. C57BL/6 (B6) male mice, 6-8 weeks old, were obtained from AnLab Co., Prague, Czech Republic. Experimental protocols were approved by the Institutional Animal Care Committee of the Institute of Molecular Genetics, Prague.

Cell lines. For the experiments, two murine models of HPV16associated tumours were used. The TC-1 tumour cell line (MHC class I-positive, non-metastasizing) was established by transformation of primary B6 mouse lung cells with HPV16 E6/E7 and activated Ha-ras DNA (32). MK16/1/ IIIABC (MK16) tumour cell line (MHC class I-deficient, spontaneously metastasizing into the lung after s.c. injection) was developed by co-transfection of murine B6 kidney cells with a mixture of activated Ha-ras oncogene and HPV16 E6/E7 genes (33). For vaccination, selected cloned IL-12producing cell subline TC-1-IL-12 (231/clone 15) obtained from Dr M. Šmahel was used. In vitro production of IL-12

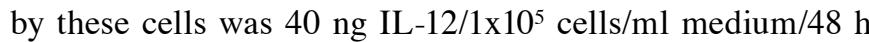
(5). All tumour cell lines were maintained in RPMI-1640 medium supplemented with $10 \%$ FCS, 2 mM L-glutamine, penicillin, streptomycin, and cultured at $37^{\circ} \mathrm{C}$ in a humidified atmosphere with $5 \% \mathrm{CO}_{2}$.

GEM treatment. For therapeutic experiments, intraperitoneal administration of $120 \mathrm{mg} / \mathrm{kg}$ of GEM (Gemzar, Eli Lilly, Indianapolis, IL) was used. The dose was established as suitable for combined treatment after performing the experiment in which B6 mice that were inoculated s.c. with $5 \times 10^{4} \mathrm{TC}-1$ cells and after approximately 15 days, when the tumours reached a size of $2-3 \mathrm{~mm}$ in diameter, were treated with different doses of GEM $(60,120$ and $240 \mathrm{mg} / \mathrm{kg}$ i.p.). The dose of $120 \mathrm{mg} / \mathrm{kg}$ was selected as suboptimal and therefore suitable for combined chemoimmunotherapy (Fig. 1A). To analyse the effect of GEM treatment on the percentage of $\mathrm{CD}_{11} \mathrm{~b}^{+} / \mathrm{Gr}-1^{+}$cells representing MDSC population, mice (three mice per group) bearing large TC-1 tumours (approximate size of $1.5 \mathrm{~cm}$ in diameter) were i.p. injected with GEM $\left(120 \mathrm{mg} / \mathrm{kg}\right.$ i.p.). Three days later, the percentage of $\mathrm{CD} 1 \mathrm{~b}^{+} /$ $\mathrm{Gr}-1^{+}$cells in the spleens of mice with tumour was analysed.
IL-12 gene therapy of TC-1 and MK16 minimal residual disease after chemotherapy with GEM. To obtain the minimal residual disease after chemotherapy, B6 mice (8 per group) were inoculated s.c. with $5 \times 10^{4} \mathrm{TC}-1$ or $1 \times 10^{5}$ MK16 tumour cells. After 15 days, when the tumours reached a size of $2-3 \mathrm{~mm}$ in diameter, the mice were randomly divided into 4 experimental groups. One experimental group was left without treatment as a control group (untreated mice). Two experimental groups of mice were injected i.p. with $120 \mathrm{mg} / \mathrm{kg}$ of GEM. A week later, one experimental group of mice treated with GEM was injected with a single dose of $15 \times 10^{6} \mathrm{IL}-12$-producing, irradiated (150 Gy), TC-1-IL-12 cells in the vicinity of the s.c. tumours. One experimental group of mice was left as GEM-only treatment and one experimental group of mice was injected with TC-1-IL-12 cells only. The growing tumours were checked twice a week. In case of metastasizing MK16 tumour, on day 57, when the mice became moribund, mice were sacrificed and autopsied for the lung metastases. The experiments were repeated three times and reproducible results are illustrated. In control experiments, the TC-1 cells without inserted gene were used without any effect (data not shown).

IL-12 gene therapy of MK16 residual disease after surgery. To obtain the minimal residual tumour disease after surgery, B6 mice were inoculated s.c. with $5 \times 10^{5}$ MK16 cells on day 0 . After 30 days, when the transplanted tumours reached $\sim 8-12 \mathrm{~mm}$ in diameter, the tumours were excised under i.p. anaesthesia, leaving no macroscopically visible tumour residuum (34). The hypothetical microscopic tumour residua after surgery were designated as surgical minimal residual tumour disease. Mice were randomly divided into 4 experimental groups (10 mice per group). One experimental group was left without treatment as a control group (operated-only mice). Two groups of experimental mice were treated with GEM (on day 1 and 7 after the surgery, $120 \mathrm{mg} / \mathrm{kg}$ i.p.). One experimental group previously treated with GEM was injected 3 days after the surgery) into the site of previous surgery with a single dose of $15 \times 10^{6} \mathrm{IL}-12$-producing, irradiated (150 Gy), TC-1-IL-12 cells. One experimental group of mice was left as GEM-only treated and the other experimental group of mice was injected with TC-1-IL-12 cells only. Seven days after the surgery, three mice from each group were sacrificed and their splenocytes were tested for IFN $\gamma$ production (ELISPOT, ELISA). The percentage of $\mathrm{CD} 11 \mathrm{~b}^{+} / \mathrm{Gr}-1^{+}$cells in the spleens was evaluated by FACS analysis. On day 40 after the operation, the mice were sacrificed and autopsied for lung metastases. The experiments were repeated three times and the representative results are illustrated.

Tumour metastases. Lungs were removed and inspected for the presence of macroscopically detectable metastases. The number of metastatic nodules was determined after fixation in Bouin's solution using a stereoscopic microscope (Stemi 2000, Carl Zeiss Jena GmbH, Jena, Germany). For histological verification of the metastases, lungs were fixed with $10 \%$ neutral buffer formalin, embedded in paraffin, and tissue sections were stained with haematoxylin and eosin (31).

ELISA. For ELISA, the supernatants obtained after $48 \mathrm{~h}$ cultivation of splenocytes $\left(2 \times 10^{6} / \mathrm{ml}\right)$ were frozen. The ELISA 
(A)

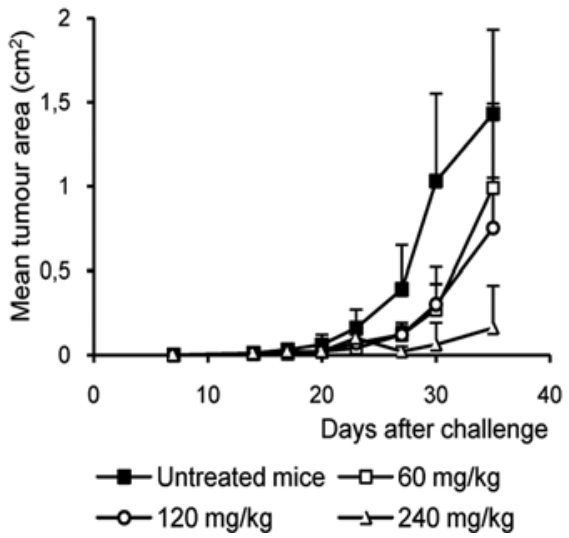

(B)

Mice bearing large TC-1 tumour

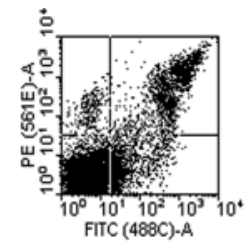

$2,2 / 13,5$

$68,1 / 16,1$

\section{Mice bearing large TC-1 tumour treated with GEM}

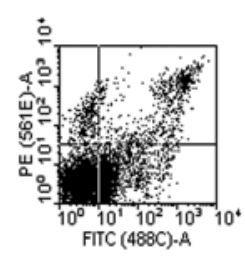

$2,7 / 5,9$

$66,6 / 24,7$

Figure 1. The effects of GEM on the growth of TC-1 tumours and on the percentage of MDSC in the spleen of mice bearing TC-1 tumours. (A) The effect of 60,120 and $250 \mathrm{mg} / \mathrm{kg}$ of GEM on the growth of the TC-1 tumours. Untreated mice vs. GEM $60 \mathrm{mg} / \mathrm{kg}$ P $>0.05$; untreated mice vs. $120 \mathrm{mg} / \mathrm{kg}$ P $<0.05$; untreated mice vs. GEM $240 \mathrm{mg} / \mathrm{kg}$ P $<0.01$; (ANOVA). (B) The effect of GEM $(120 \mathrm{mg} / \mathrm{kg}$ i.p.) on the percentage of MDSC in the spleen of mice bearing the growing TC-1 tumours. GEM decreases percentage of $\mathrm{CD} 11 \mathrm{~b}^{+}(\mathrm{FITC}) / \mathrm{Gr}-1^{+}(\mathrm{PE})$ cells (13.5\% without GEM treatment vs. $5.9 \%$ after GEM treatment).

kit (BD Biosciences, San Diego, CA) was used according to the manufacturer's instructions.

ELISPOT assay. To determine the portion of IFN $\gamma$-secreting spleen cells, an ELISPOT kit for detection of murine IFN $\gamma$ (BD PharMingen, San Diego, CA) was used. The splenocytes were cultured for $48 \mathrm{~h}$ and then placed to the wells of ELISPOT plates (concentration $5 \times 10^{5}$ cells/well) for $24 \mathrm{~h}$. The plates were then processed according to the manufacturer's instructions (BD PharMingen). Coloured spots were counted with CTL Analyzer LLC (CTL, Cleveland, OH) and analyzed using the ImmunoSpot Image Analyzer software.

Flow cytometry (FACS). To determine the percentage of $\mathrm{CD}_{11 \mathrm{~b}}{ }^{-} \mathrm{Gr}-1^{+}$cells, FITC rat anti-mouse CD11b (M1/70) and PE rat anti-mouse Ly-6G and Ly-6C (Gr-1) (RB6-8C5) were used. As isotype controls, FITC and PE-labelled antibody of irrelevant specificity were used. All products were purchased from BD PharMingen. Flow cytometry was performed using LSR II flow cytometer (BD Biosciences, San Jose, CA).

Statistical analyses. For statistical analyses, Student's t-test and $\chi^{2}$ comparison test were used. Analysis of variance (ANOVA) from the NCSS, Number Cruncher Statistical System (Kaysville, UT) statistical package was used.

\section{Results}

GEM treatment. To select the convenient dose of GEM for therapeutic experiments, B6 mice were inoculated subcutaneously (s.c.) with $5 \times 10^{4}$ TC- 1 cells and after 15 days, when the tumours reached a size of $2-3 \mathrm{~mm}$ in diameter, the mice were intraperitoneally (i.p.) treated with different doses of GEM: 60,120 and $240 \mathrm{mg} / \mathrm{kg}$. The dose of $120 \mathrm{mg} / \mathrm{kg}$ was selected as suboptimal and therefore suitable for chemoimmunotherapy (Fig. 1A).

To analyse the effect of this selected dose of GEM on the percentage of $\mathrm{CD}_{11 \mathrm{~b}} / \mathrm{Gr}-1^{+}$cells in spleen, the mice $(3$ mice per group) bearing large TC-1 tumours (approximate size of $1.5 \mathrm{~cm}$ in diameter) were i.p. injected with GEM $(120 \mathrm{mg} /$ $\mathrm{kg}$ i.p.). Three days later, the percentage of $\mathrm{CD} 11 \mathrm{~b}^{+} / \mathrm{Gr}-1^{+}$ cells in the spleen of the mice with tumour was analysed. The administration of GEM decreased the percentage of $\mathrm{CD}_{11 \mathrm{~b}^{+}}$ Gr- $1^{+}$cells in the spleens (13.5\% without GEM treatment vs. $5.9 \%$ after GEM treatment) (Fig. 1B). The impact of GEM

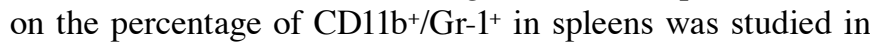
mice bearing large tumours since its percentage in mice from all experimental groups three days after TC-1-IL-12 vaccine administration was not significantly different from the values observed in the healthy mice (data not shown).

\section{IL-12 gene therapy of TC-1 and MK16 minimal residual} disease after chemotherapy with GEM. Mice were inoculated s.c. with $5 \times 10^{4} \mathrm{TC}-1$ cells or $1 \times 10^{5}$ MK16 cells. On day 15 , when the tumours reached a palpable size of $2-3 \mathrm{~mm}$ in diameter, the tumour-bearing mice were randomly divided into three experimental groups and one control group (untreated mice). Two experimental groups were injected i.p. with the dose of $120 \mathrm{mg} / \mathrm{kg}$ GEM. Seven days after chemotherapy, the first experimental group was left as mice treated with GEM only and the second experimental group previously treated with GEM was injected peritumorally with a single dose of the IL-12-producing vaccine. The third experimental group was treated with the IL-12-producing vaccine only. As can be seen in Fig. 2A, the treatment of TC-1 tumours with GEM resulted in a substantial tumour inhibition in comparison with the untreated controls. This effect was further significantly potentiated by the therapy with the IL-12-producing cells. The treatment of the MK16 tumours with GEM resulted also in the substantial tumour growth inhibition in comparison with the untreated controls. The treatment with the TC-1-IL-12 vaccine alone led to non-significant increase of tumour volumes. The therapeutic effect of GEM was potentiated after combination with IL-12-producing cells, however, the difference between GEM-treated only mice and combination of GEM plus TC-1-IL-12 was not significant (Fig. 2B). In autopsy, 75\% of the untreated mice exhibited lung metastases. Combined treatment of the MK16 residual disease with GEM plus IL-12-producing vaccine substantially reduced the percentage of mice with lung metastases up to $29 \%$ and also significantly 


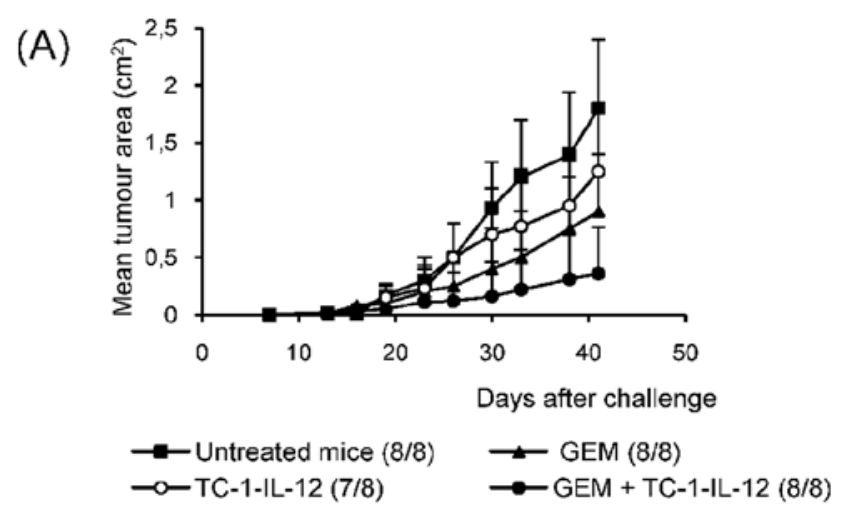

(B)

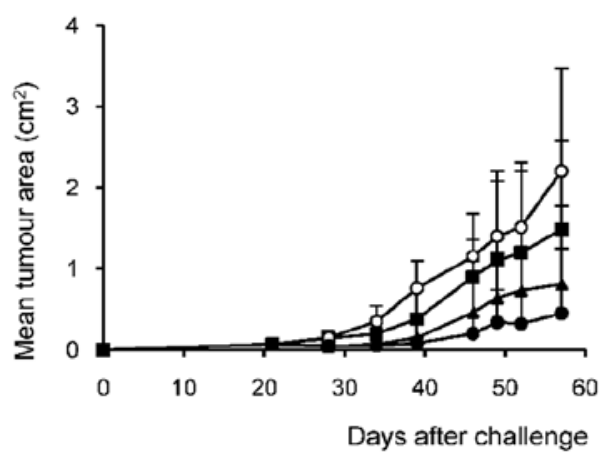

(C)

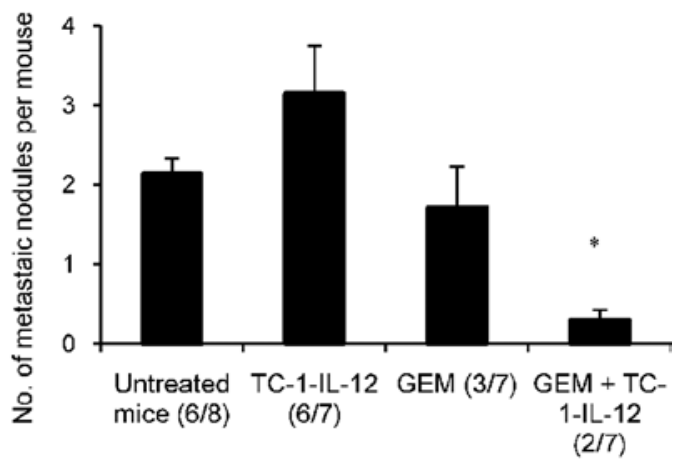

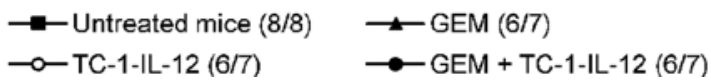

Figure 2. Treatment of the residual tumour disease after chemotherapy of TC-1 and MK16 tumours. (A) The effect of peritumoral administration of the IL-12producing vaccine on TC-1 tumour residua after GEM treatment. Untreated mice vs. GEM P<0.05; untreated mice vs. GEM + TC-1-IL-12 P<0.01; GEM vs. GEM + TC-1-IL-12 P<0.05; TC-1-IL-12 vs. GEM + TC-1-IL-12 P<0.05 (ANOVA). Numbers in parentheses represent numbers of mice with tumour/number of mice in experimental group. (B) The effect of peritumoral administration of IL-12-producing vaccines on growing MK16 tumour residua after GEM treatment. Untreated mice vs. GEM, GEM + TC-1-IL-12 P<0.05; TC-1-IL-12 vs. GEM, GEM + TC-1-IL-12 P<0.05 (ANOVA). Numbers in parentheses represent number of mice with tumour/numbers of mice in the experimental group. (C) The effect of peritumoral administration of IL-12-producing vaccines after GEM treatment on MK16 lung metastases. Number of mice with lung metastases: untreated mice, TC-1-IL-12 vs. GEM + TC-1-IL-12 P<0.05 ( $\left.\chi^{2}\right)$. Number of metastatic nodules per mouse: *denotes $\mathrm{P}<0.05$ compared to untreated mice, GEM, TC-1-IL-12 (Student's t-test). Numbers in parentheses represent number of mice with lung metastases/numbers of mice in the experimental group.

reduced the number of the metastatic nodules per mouse (Fig. 2C).

IL-12 gene therapy of MK16 residual disease after surgery. Mice were inoculated s.c. with $5 \times 10^{5}$ MK16 cells and when their tumours reached $8-12 \mathrm{~mm}$ in diameter, the tumours were excised and mice were divided into 4 groups. One group was left as a control group (operated-only mice). Two groups of mice were treated i.p. with GEM $120 \mathrm{mg} / \mathrm{kg}$ on days 1 and 7 after the surgery. Three days after surgery, one group of experimental mice treated with GEM was left as mice treated with GEM only and the second group previously treated with GEM was injected into the site of previous surgery with a single dose of IL-12-producing vaccine. One experimental group was similarly treated with the IL-12-producing vaccine only. As can be seen in Fig. 3A, the tumour recurrence rate of the MK16 carcinoma on day 40 after the surgery in the operated-only group and in the group treated with the IL-12producing vaccine only was $60 \%$ and in the group treated with GEM it was $40 \%$. The combined treatment of the MK16 residual tumour disease with GEM and IL-12-producing vaccine completely inhibited the growth of recurrent tumours.
In autopsy, $50 \%$ of the operated-only mice and mice treated with the IL-12-producing vaccine only exhibited lung metastases. The combined treatment of MK16 residual disease with GEM plus IL-12-producing vaccine significantly reduced the percentage of mice with lung metastases to $10 \%$. The number of the metastatic nodules per mouse was also significantly reduced (Fig. 3B.)

Analysis of immune responses. Seven days after surgery, the spleens from three mice of each experimental group were tested for the amount of IFN $\gamma$-secreting cells (ELISPOT), IFN $\gamma$ production (ELISA) and also for the percentage of $\mathrm{CD} 11 \mathrm{~b}^{+} / \mathrm{Gr}-1^{+}$cells. The percentage of the IFN $\gamma$-producing cells after the treatment with GEM, IL-12-producing vaccine and their combination was significantly increased as compared to the operated-only group. The highest percentage was found in the group treated with GEM plus IL-12-producing cells (Fig. 3C). In accordance with the results of the ELISPOT assay, the treatment with GEM and subsequent immunotherapy with the IL-12-producing cells led to a significant increase of the IFN $\gamma$ production by splenocytes detected by ELISA in supernatants of cultured splenocytes (Fig. 3D). 
(A)
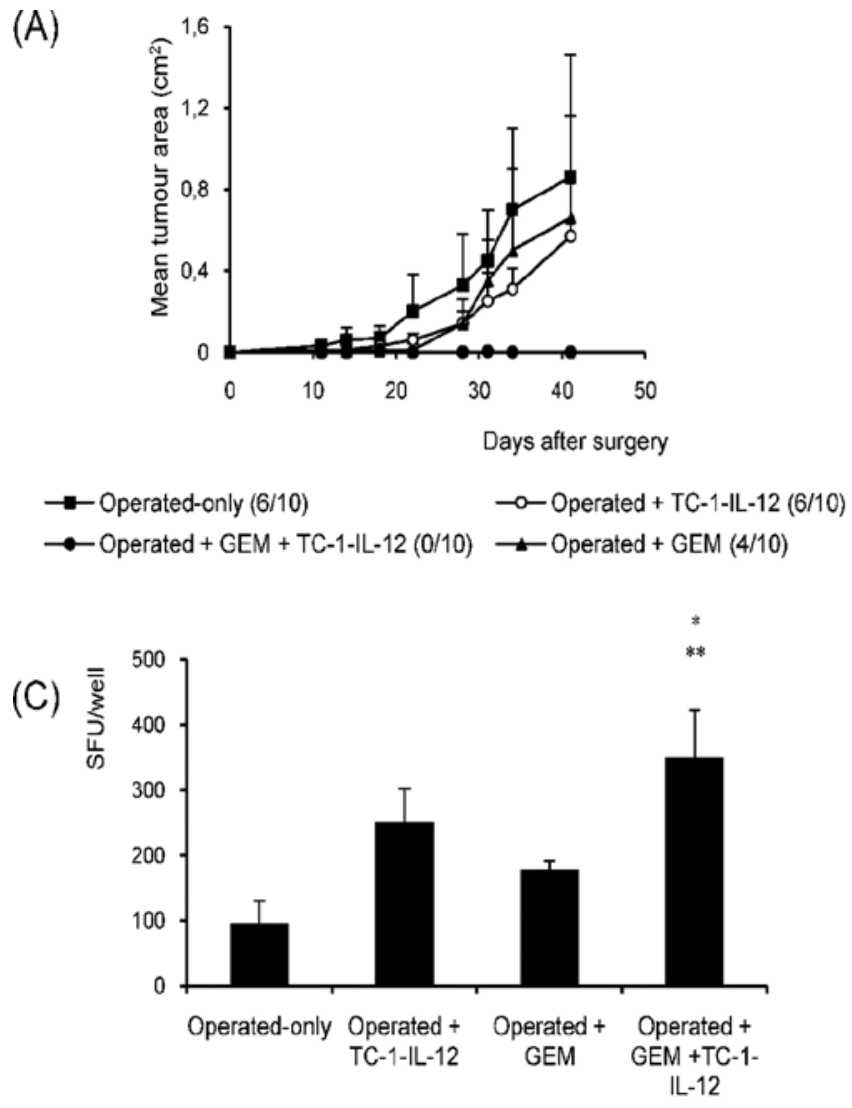

(B)

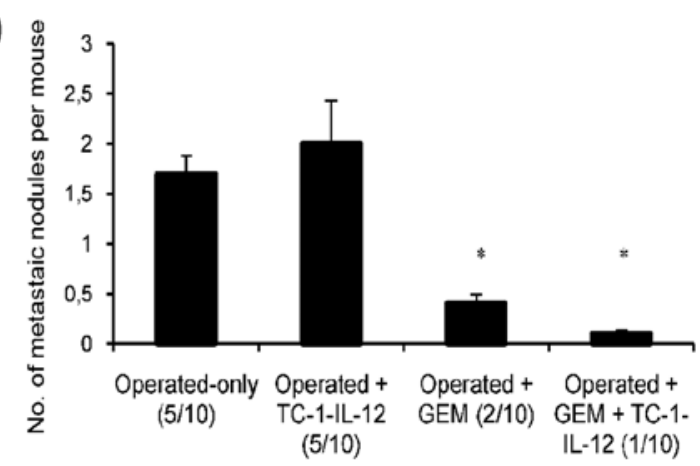

Figure 3. Treatment of residual tumour disease after surgery of MK16 tumours. (A) The effect of combined treatment with GEM plus IL-12-producing vaccine on MK16 tumour residua after surgery. Number of mice with tumour recurrences: operated-only, TC-1-IL-12 vs. TC-1-IL-12 + GEM P<0.01; GEM vs.TC-1-IL-12 + GEM P<0.05 $\left(\chi^{2}\right)$. Growth curves: operated-only, GEM, TC-1-IL-12 vs. TC-1-IL-12 + GEM P<0.05 (ANOVA). Numbers in parentheses represent numbers of mice with tumour recurrences/number of mice in the experimental group. (B) The effect of combined treatment with GEM plus IL-12producing vaccine on MK16 lung metastases. Number of mice with lung metastases: operated-only, TC-1-IL-12 vs. GEM + TC-1-IL-12 P<0.05 ( $\left.\chi^{2}\right)$. Number of metastatic nodules per mouse: *denotes $\mathrm{P}<0.05$ compared to operated-only, TC-1-IL-12 (Student's t-test). Numbers in parentheses represent numbers of mice with lung metastases/number of mice in the experimental group. (C and D) Analysis of the IFN $\gamma$ production by splenocytes: ELISPOT assay (C) and ELISA (D). The highest IFN $\gamma$ production was found in the spleens of mice treated with GEM plus IL-12-producing vaccine. *Denotes $\mathrm{P}<0.05$ compared to operated-only; **denotes $\mathrm{P}<0.05$ compared to GEM; ${ }^{* * *}$ denotes $\mathrm{P}<0.05$ compared to operated-only, TC-1-IL-12, GEM (Student's t-test).

\section{Discussion}

In this study we have demonstrated that local administration of the genetically modified, IL-12-producing tumour vaccine can augment the chemotherapy of HPV 16-associated murine tumours with cytostatic agent GEM when GEM was used in suboptimal therapeutic doses. The objective was to suggest a therapeutic setting of combined chemoimmunotherapy, utilizing the GEM immunomodulatory capacity, that can be based on lower doses of the chemotherapeutic agent and thus induce less serious adverse effects as compared to the monotherapy.

Residual tumour disease after chemotherapy or surgery represents one of the most favourable targets for adjuvant vaccination and immunotherapy because of the low tumour burden at the time of the immunotherapy and because of pre-sensitization of the host by the relevant tumour antigens during the temporary tumour growth prior to the chemotherapy and surgery.

Local peritumoral gene therapy with IL-12-producing tumour cellular vaccine has been selected for the combined chemoimmunotherapy with GEM since IL-12 is one of the most effective immunostimulatory cytokine with antitumor effects and its systemic administration is associated with serious systemic toxicity. In our previous experiments, the efficacy of the cellular vaccines producing IL-12 in the treatment of residual disease after cytoreductive chemotherapy with another immunomodulatory cytostatic agent cyclophosphamide was demonstrated (5-7,31).

In the TC-1 tumour model, local administration of irradiated tumour vaccine carrying an inserted IL-12 gene and constitutively producing IL-12 enhanced the effect of intraperitoneal cytoreductive therapy with GEM. Unlike for the TC-1 tumours, the beneficial effects of the combination of GEM and IL-12-producing vaccine were not significant in the MK16 model. These results could be explained by our previous finding that the MK16 tumour is only moderately immunogenic and less sensitive to the immunotherapy than immunogenic TC-1 tumour (35), e.g. due to the MHC class I downregulation on the surface of the MK16 cells. However, significant improvement of the therapeutic efficacy was demonstrated when the anti-metastatic effects of this combinational treatment were evaluated in MK16 model.

Further, minimal residual disease of MK16 tumour after surgery, which was shown to be sensitive to immunotherapy in our previous study (31), was chosen. In minimal residual 
disease of MK16 tumour after surgery, a significant antitumour and anti-metastatic effect of the combination of GEM plus IL-12-producing cellular vaccine combination was demonstrated. As compared to the therapy of growing MK16 tumours, the synergistic effects of the combined treatment of the minimal residual tumour disease after surgery were more pronounced and no recurrent tumours after the combined treatment were observed.

The therapeutic efficacy of particular treatments in general correlated with the level of immune response. Indeed, the highest percentage of IFN $\gamma$-producing cells (ELISPOT) and the highest IFN $\gamma$ production by splenocytes (ELISA) was found in the group treated with GEM plus IL-12-producing cells, although both monoterapies with GEM or IL-12producing cells also resulted in significant increase of the IFN $\gamma$ production. The percentage of the $\mathrm{CD}_{11 \mathrm{~b}^{+} / \mathrm{Gr}-1^{+} \text {cells }}$ three days after the TC-1-IL-12 vaccine administration was examined and it was not significantly different in all experimental and control groups from the values observed in healthy mice. As it was shown in mice bearing large tumours, selected GEM concentration $(120 \mathrm{mg} / \mathrm{kg})$ was proved to reduce MDSC cells, so it is plausible that its anti-suppressive effects contributed to increased immune response.

The experiments were performed on two HPV16-associated tumour models (on TC-1 tumour cells that are MHC class I-positive, and on MK16 tumour cells that are MHC class I-deficient) with similar therapeutic results. It has been demonstrated previously that the MK16 cells, when cultivated in vitro in the presence of IFN $\gamma$, can acquire, together with the expression of MHC class I molecules, the sensitivity to the cytolytic effect of splenocytes from the MK16 tumourimmunized mice (35). The up-regulation was also observed in vivo in the recurrences after surgery and it has reached the level required for in vitro cytolysis of the tumour cells by CTLs (35). Co-operation of the MHC-restricted and MHC non-restricted immune mechanisms thus could contribute to the therapeutic effects of IL-12 both in the TC-1 and MK16 model.

In this communication we have demonstrated for the first time that the local administration of irradiated tumour vaccine constitutively producing IL-12 enhanced the effect of intraperitoneal cytoreductive therapy with the immunomodulatory cytostatic agent GEM in two experimental tumour models with distinct MHC class I cell surface expression levels. Our results have shown that the combination of immunotherapy with IL-12 and chemotherapy with GEM could be a promising strategy. If these findings can be confirmed in additional experimental and human tumours, they may have important implications for the development of immunotherapeutic strategies.

\section{Acknowledgements}

This study was supported by grants No. 301/09/1024 and No. 301/07/1410 from the Grant Agency of the Czech Republic, by grant of the Clinigene project EU-FP6-NOE No. 018933, and, in part, by grant No. AVOZ50520514 awarded by the Academy of Sciences of the Czech Republic. The authors are grateful to Professor Vladimír Vonka for the kind gift of MK16 cells, to Dr Michal Šmahel for the kind gift of TC-1-
IL-12 cells, to Mrs. Renata Turečková and Marie Malečková for skilful technical assistance, and to Dr Šárka Takačová for editorial help.

\section{References}

1. Nowak AK, Lake RA and Robinson BW: Combined chemoimmunotherapy of solid tumours: improving vaccines? Adv Drug Deliv Re 1: 975-990, 2006.

2. Ramakrishnan R, Antonia S and Gabrilovich DI: Combined modality immunotherapy and chemotherapy: a new perspective. Cancer Immunol Immunother 57: 1523-1529, 2008.

3. Nowak AK, Lake RA, Marzo AL, Scott B, Heath WR, Collins EJ, Frelinger JA and Robinson BW: Induction of tumor cell apoptosis in vivo increases tumor antigen cross-presentation, cross-priming rather than cross-tolerizing host tumor-specific CD8 T cells. J Immunol 170: 4905-4913, 2003.

4. Bubeník J: Genetically modified vaccines augment the efficacy of cancer surgery and chemotherapy. Folia Biol (Praha) 55: 199-200, 2009.

5. Indrova M, Bieblova J, Jandlova T, Vonka V, Pajtasz-Piasecka E and Reinis M: Chemotherapy, IL-12 gene therapy and combined adjuvant therapy of HPV16-associated MHC class I-proficient and -deficient tumours. Int J Oncol 28: 253-259, 2006.

6. Indrová M, Bubeník J, Mikysková R, Mendoza L, Símová J, Bieblová J, Jandlová T, Jinoch P, Smahel M, Vonka V and Pajtasz-Piasecka E: Chemoimmunotherapy in mice carrying HPV-16 associated, MHC class I and MHC class I tumours: effects of CBM-4A potentiated with IL-2, IL-12, GM-CSF and genetically modified tumour vaccines. Int J Oncol 22: 691-695, 2003.

7. Indrova M, Bieblova J, Bubenik J and Reinis M: IL-12 immunotherapy of minimal residual disease in murine models of HPV16-associated tumours: induction of immune responses, cytokine production and kinetics of immune cell subsets. Int $\mathrm{J}$ Oncol 32: 499-507, 2008.

8. Bubeník J and Šímová J: Genetically modified cellular vaccines against human papillomavirus type 16 (HPV16)-associated tumors: adjuvant treatment of minimal residual disease after surgery/chemotherapy. J BUON 14: 169-173, 2009.

9. Ménard C, Martin F, Apetoh L, Bouyer F and Ghiringhelli F: Cancer chemotherapy: not only a direct cytotoxic effect, but also an adjuvant for antitumor immunity. Cancer Immunol Immunother 57: 1579-1587, 2008.

10. Nowak AK, Robinson BW and Lake RA: Gemcitabine exerts a selective effect on the humoral immune response: implications for combination chemo-immunotherapy. Cancer Res 62: 2353-2358, 2002.

11. Nowak AK, Robinson BW and Lake RA: Synergy between chemotherapy and immunotherapy in the treatment of established murine solid tumors. Cancer Res 63: 4490-4496, 2003.

12. Machiels JP, Reilly RT, Emens LA, Ercolini AM, Lei RY, Weintraub D, Okoye FI and Jaffee EM: Cyclophosphamide, doxorubicin, and paclitaxel enhance the antitumor immune response of granulocyte/macrophage-colony stimulating factorsecreting whole-cell vaccines in HER-2/neu tolerized mice. Cancer Res 61: 3689-3697, 2001.

13. Malvicini M, Rizzo M, Alaniz L, Piñero F, García M, Atorrasagasti C, Aquino JB, Rozados V, Scharovsky OG, Matar P and Mazzolini G: A novel synergistic combination of cyclophosphamide and gene transfer of interleukin-12 eradicates colorectal carcinoma in mice. Clin Cancer Res 15: 7256-7265, 2009.

14. Hui YF and Reitz J: Gemcitabine: a cytidine analogue active against solid tumors. Am J Health Syst Pharm 54: 162-170, 1997.

15. Suzuki E, Sun J, Kapoor V, Jassar AS and Albelda SM: Gemcitabine has significant immunomodulatory activity in murine models independent of its cytotoxic effects. Cancer Biol Ther 6: 880-885, 2007.

16. Toschi L, Finocchiaro G, Bartolini S, Gioia V and Cappuzzo F: Role of gemcitabine in cancer therapy. Future Oncol 1: 7-17, 2005.

17. Plate JM, Plate AE, Shott S, Bograd S and Harris JE: Effect of gemcitabine on immune cells in subjects with adenocarcinoma of the pancreas. Cancer Immunol Immunother 54: 915-925, 2005. 
18. Suzuki E, Sun J, Kapoor V, Jassar AS, Kaiser LR and Albelda SM: Gemcitabine selectively eliminates splenic $\mathrm{gr}-1^{+}$

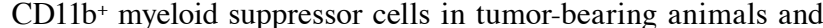
enhances antitumor immune activity. Clin Cancer Res 11: 6713$6721,2005$.

19. Liu WM, Fowler DW, Smith P and Dalgleish AG: Pre-treatment with chemotherapy can enhance the antigenicity and immunogenicity of tumours by promoting adaptive immune responses. Br J Cancer 102: 115-123, 2010.

20. Le HK, Graham L, Cha E, Morales JK, Manjili MH and Bear HD: Gemcitabine directly inhibits myeloid derived suppressor cells in BALB/c mice bearing $4 \mathrm{~T} 1$ mammary carcinoma and augments expansion of $\mathrm{T}$ cells from tumorbearing mice. Int Immunopharmacol 9: 900-909, 2009.

21. Sinha P, Clements VK, Bunt SK, Albelda SM and OstrandRosenberg S: Cross-talk between myeloid-derived suppressor cells and macrophages subverts tumor immunity toward a type 2 response. J Immunol 179: 977-983, 2007.

22. Brunda MJ, Luistro L, Rumennik L, Wright RB, Dvorozniak M, Aglione A, Wigginton JM, Wiltrout RH, Hendrzak JA and Palleroni AV: Antitumor and antimetastatic activity of interleukin 12 against murine tumors. J Exp Med 178: 1223-1230, 1993.

23. Nanni P, Rossi I, De Giovanni C, Landuzzi L, Nicoletti G, Stoppacciaro A, Parenza M, Colombo MP and Lollini PL: Interleukin 12 gene therapy of MHC-negative murine melanoma metastases. Cancer Res 58: 1225-1230, 1998.

24. Vagliani M, Rodolfo M, Cavallo F, Parenza M, Melani C, Parmiani G, Forni G and Colombo MP: Interleukin 12 potentiates the curative effect of a vaccine based on interleukin 2-transduced tumor cells. Cancer Res 56: 467-470, 1996.

25. Cavallo F, Signorelli P, Giovarelli M, Musiani P, Modesti A, Brunda MJ, Colombo MP and Forni G: Antitumor efficacy of adenocarcinoma cells engineered to produce interleukin 12 (IL-12) or other cytokines compared with exogenous IL-12. J Natl Cancer Inst 89: 1049-1058, 1997.

26. Bubenik J: Genetically modified cellular vaccines for therapy of human papilloma virus type 16 (HPV 16)-associated tumours. Curr Cancer Drug Targets 8: 180-186, 2008.
27. Portielje JE, Gratama JW, van Ojik HH, Stoter G and Kruit WH: IL-12: a promising adjuvant for cancer vaccination (review). Cancer Immunol Immunother 52: 133-144, 2003

28. Colombo MP and Trinchieri G: Interleukin-12 in anti-tumor immunity and immunotherapy. Cytokine Growth Factor Rev 13: 155-168, 2002.

29. Indrová M, Bieblová J, Rossowska J, Kuropka P, PajtaszPiasecka E, Bubeník J and Reinis M: HPV 16-associated tumours: IL-12 can repair the absence of cytotoxic and proliferative responses of tumour infiltrating cells after chemotherapy. Int J Oncol 34: 173-179, 2009.

30. Imagawa $\mathrm{Y}$, Satake $\mathrm{K}$, Kato $\mathrm{Y}$, Tahara $\mathrm{H}$ and Tsukuda $\mathrm{M}$ : Antitumor and antiangiogenic effects of interleukin 12 gene therapy in murine head and neck carcinoma model. Auris Nasus Larynx 31: 239-245, 2004.

31. Mikysková R, Bubenik J, Mendoza L, Vonka V, Smahel M, Símová J and Jandlová T: Local cytokine treatment of HPV16associated tumours results in inhibition of their lung metastases. Clin Exp Metastasis 18: 581-587, 2000.

32. Lin KY, Guarnieri FG, Staveley-O'Carroll KF, Levitsky HI, August JT, Pardoll DM and Wu TC: Treatment of established tumors with a novel vaccine that enhances major histocompatibility class II presentation of tumor antigen. Cancer Res 56: 21-26, 1996.

33. Smahel M, Sima P, Ludvikova V, Marinov I, Pokorna D and Vonka V: Immunisation with modified HPV16 E7 genes against mouse oncogenic TC-1 cell sublines with downregulated expression of MHC class I molecules. Vaccine 21: 1125-1136, 2003.

34. Vlk V, Rössner P, Indrová M, Bubeník J and Sobota V: Interleukin-2 gene therapy of surgical minimal residual tumour disease. Int J Cancer 76: 115-119, 1998.

35. Mikysková R, Bubeník J, Vonka V, Smahel M, Indrova M, Bieblová J, Símová J and Jandlová T: Immune escape phenotype of HPV16-associated tumours: MHC class I expression changes during progression and therapy. Int J Oncol 26: 521-527, 2005. 